hep-th/9608147, SCIPP 96/35

\title{
Dynamical Supersymmetry Breaking versus Run-away Behavior in Supersymmetric Gauge Theories
}

\author{
Yuri Shirman* \\ Santa Cruz Institute for Particle Physics \\ University of California, Santa Cruz, CA 95064
}

\begin{abstract}
We consider Dynamical Supersymmetry Breaking (DSB) in models with classical flat directions. We analyze a number of examples, and develop a systematic approach to determine if classical flat directions are stabilized in the full quantum theory, or lead to run-away behavior. In some cases pseudo-flat directions remain even at the quantum level before taking into account corrections to the Kähler potential. We show that in certain limits these corrections are calculable. In particular, we find that in the Intriligator-Thomas $S U(2)$ and its generalizations, a potential for moduli is generated. Moreover, there is a region of the parameter space where Kähler potential corrections lead to calculable (local) minima at large but finite distance from the origin.
\end{abstract}

$8 / 96$

* yuri@scipp.ucsc.edu 


\section{Introduction}

If supersymmetry is relevant to the real world it is important to investigate mechanisms by which it could be broken. Models of dynamical supersymmetry breaking (DSB) have been known for some time [1]. Recently, many more models have been constructed [2/8]. Traditionally, one of the requirements in the construction of the models with DSB was that they not possess classical flat directions, and many more examples of this kind have been studied recently. This requirement was due to the observation [1] that when classical flat directions are lifted by non-perturbative dynamics one usually finds run-away behavior.

It was pointed out in [3] that this may not be the case if part of the gauge group remains unbroken with coupling which does not tend to zero along the classical flat direction. An example with $S U(2)$ gauge group and matter transforming as $4 S U(2)$ doublets and 6 gauge singlets (as well as generalizations for $S U(N)$ and $S P(N)$ groups) was constructed in [3,4] where effects of the strong dynamics led to non-vanishing energy along the classical flat direction. However, a pseudo-flat direction remained even in the presence of the SUSY breaking superpotential. The location of the minimum along this direction is determined by the quantum corrections to the Kähler potential. Due to the strongly coupled nature of the theory, these corrections could not be determined, leaving open the possibility that the minimum is infinitely far along the flat direction. Somewhat similar models based on dual gauge groups were constructed in [3,5]:6].

In this note we will suggest a systematic approach to the analysis of the physics along classical flat directions. We will discuss several examples, and identify properties of the models which lead to (non) stabilization of the classical flat directions. In fact it is easy to summarize our approach to the question of the possible run-away behavior. Consider a model with classical flat directions (assume for simplicity that there is a single direction described by modulus $S$ ). Can quantum effects stabilize the moduli near the origin, or there will be a run-away behavior and no stable vacuum? To answer this question let us consider the theory at large vacuum expectation value (vev) of $S$. It is convenient to split 
the scalar potential into two parts国:

$$
V=V_{L}+V_{S}
$$

where $V_{L}$ is the scalar potential of the "reduced" theory with $S$ considered as a parameter $\left(V_{L}\right.$ may or may not depend on $S$ ), and $V_{S}=\left|\frac{\partial W}{\partial S}\right|^{2}$. One should first ask whether there are solutions to the equation

$$
V_{L}=0
$$

If there are no solutions to this equation, then the energy for fixed moduli is proportional to the scale characterizing the "reduced" theory, $V \sim \Lambda_{L}^{4}$. If $\Lambda_{L}$ grows with $S$, then there is an effective potential for the modulus, and no run-away behavior. This does not yet mean that supersymmetry is broken in the full theory - to establish that one needs to analyze the theory near the origin of the moduli space. We will describe an example of such a model in $\S 5$. Another logical possibility is that $\Lambda_{L} \rightarrow 0$ when $S \rightarrow \infty$. If the same is true for $V_{S}$, then there is a run-away direction in the full theory.

In the following sections we will be mostly considering models such that (1.2) will have a solution for every value of $S$. In such models the question of run-away behavior will become a question about the behavior of $V_{S}$ for large $S$ (subject to (1.2)). We will discuss examples in which run-away directions persist in the full theory as well as examples where classical flat directions are stabilized sufficiently far from the origin. We will also consider an example where moduli are stabilized near the origin, and analysis in the region of the strong coupling is required to establish SUSY breaking.

\section{SUSY QCD with singlets}

We will begin by considering massive SUSY QCD with $N_{f}=N_{c}$. It is known that in this model the classical moduli space is modified quantum mechanically [9]:

$$
\operatorname{det}(M)-B \bar{B}=\Lambda^{2 N}
$$

1 This can be done either in the full theory or in the low energy effective theory applicable for large $S$. 
where $M_{i j}=Q_{i} \bar{Q}_{j}, B=Q^{N}, \bar{B}=\bar{Q}^{N}$. This constraint can be enforced by a Lagrange multiplier term, so that the full superpotential is:

$$
W=A\left(\operatorname{det} M-B \bar{B}-\Lambda^{2 N}\right)+m_{i j} M_{i j}
$$

In the vacuum mesons have the following vev's:

$$
M_{i j}=\left(\operatorname{det}(m) \Lambda^{2 N}\right)^{\frac{1}{N}}\left(\frac{1}{m}\right)_{i j}^{-1}
$$

It was found in [3, 团 that a simple modification of this model breaks supersymmetry dynamically. Instead of giving mass to the quarks one can couple them to $N^{2}+2$ gauge singlets:

$$
W_{\text {tree }}=\sum_{i j}^{N} \lambda_{i j} S_{i j} M_{i j}+X_{1} B+X_{2} \bar{B} .
$$

This superpotential lifts all flat directions associated with $\mathrm{SU}(\mathrm{N})$, but classically there are still flat directions for the singlets. Quantum mechanically the energy is non-vanishing along these flat directions [3]. When the singlets have large vev's quarks become massive and the low energy theory is pure Yang-Mills with the scale

$$
\Lambda_{Y M}^{3 N}=\operatorname{det}(\lambda S) \Lambda^{2 N}
$$

Gaugino condensation gives rise to a superpotential

$$
W=\Lambda_{Y M}^{3}=\left(\operatorname{det}(\lambda S) \Lambda^{2 N}\right)^{\frac{1}{N}} \sim \lambda S \Lambda^{2}
$$

and the energy does not vanish in the limit $S \rightarrow \infty$. Classically there is a supersymmetric minimum at the origin of the $S U(N)$ moduli space. This, however, is not compatible [3,4] with the quantum constraint (2.1). To see this let us split scalar potential into two terms

$$
V=V_{L}+V_{S}=\left(\left|\frac{\partial W}{\partial A}\right|^{2}+\sum_{i j}\left|\frac{\partial W}{\partial M_{i j}}\right|^{2}\right)+\sum_{i j}\left|\frac{\partial W}{\partial S_{i j}}\right|^{2}
$$

Here the $V_{L}$ is just the scalar potential of SQCD with singlet vev's playing the role of masses. It vanishes whenever (2.3) is satisfied. The second term, however, contains a sum over all fields involved in the quantum constraint (2.1), and therefore can not vanish. 
The potential of eqn. (2.7) is independent of $S$. Therefore, at the level of the analysis performed so far there is still an exact flat direction even though supersymmetry is broken. In [3] various possibilities for the behavior of the theory at large $S$ were considered, including the existence of the minimum for $S \rightarrow \infty$. However, one can easily see that this is not the case. We will consider $S U(2)$ with 4 doublets and 6 singlets as an example. For simplicity, take the tree level superpotential to possess global $S U(4)$ symmetry:

$$
W_{\text {tree }}=\lambda \sum_{i<j}^{4} S_{i j} Q_{i} Q_{j}
$$

Note that the energy is proportional to the coupling constant squared $|\lambda|^{2} . \lambda$ should be interpreted as the running coupling constant, $\lambda(S)$. (More precisely, it is $\lambda(\lambda S)$. This can be determined by, for example, carefully implementing scale matching prescription (2.5) so that analyticity of the gauge coupling function is preserved at the two loop level.) The $S$ dependence of the coupling constant corresponds to the renormalization of the Kähler potential. For large $S$ and small $\lambda$ a perturbative calculation is valid and gives

$$
\beta_{\lambda}=\frac{8}{16 \pi^{2}} \lambda^{3}-\frac{3}{16 \pi^{2}} \lambda g^{2} .
$$

If $\lambda$ is taken sufficiently large, the right-hand side of (2.9) is always positive, and the coupling constant $\lambda$ is not asymptotically free. This corresponds to a growing potential for $S$, and the minimum is necessarily near the origin 2 (its exact location can not be determined by this method). A somewhat more interesting situation arises if we choose the coupling constant so that at a certain scale $M \gg \Lambda$ the right-hand side of (2.9) vanishes. Note that $\frac{\partial(\lambda / g)}{\partial t}>0$ in this model, and there is no fixed point for the Yukawa coupling constant. As a result $\lambda$ exhibits an asymptotically free behavior below $M$, and grows above $M$. In such a regime scalar the potential of the model has a minimum at $S \sim \frac{M}{\lambda}$.

Now, let us consider one more modification of the model, namely, suppose that only $N-1$ flavors are massive while the last flavor couples to the gauge singlet

$$
W_{\text {tree }}=\sum_{i j}^{N-1} m_{i j} M_{i j}+S M_{N N} .
$$

2 This description breaks down at large scales as $\lambda$ hits its Landau singularity. At those scales an underlying microscopic description of the theory should be used. However, there still exists (at least a local) minimum near the origin. 
Splitting the scalar potential into two terms in analogy with (2.7) we see that $V_{S}=\left|M_{N N}\right|^{2}$. This can be infinitesimally small with some other mesons having infinitely large vev's in such a way that both $V_{L}=0$, and the quantum constraint (2.1) is satisfied. From (2.3) we find 3 :

$$
M_{i j} \sim S^{\frac{1}{N}}, \quad M_{N N} \sim S^{-\frac{N-1}{N}}
$$

where $i, j=1 \ldots N-1$. We see that SUSY is restored when $S \rightarrow \infty$. One can reach the same conclusion in any modification of the $S U(N)$ theory with $N$ flavors where some of the classical flat directions are lifted by the mass terms rather than by coupling to gauge singlet fields.

Before going on to the next section, where we will use these results, let us briefly mention analogous results for the model based on $S P(N)$ gauge group with $N+1$ flavors in the fundamental representation. The quantum modified constraint is given by [10]:

$$
P f(M)=\Lambda^{2(N+1)},
$$

where $M_{i j}=Q_{i} Q_{j}$. In the massive case the vacuum is given by:

$$
M_{i j}=\left(\operatorname{Pf}(m) \Lambda^{2(N+1)}\right)^{\frac{1}{N+1}}\left(\frac{1}{m}\right)_{i j}^{-1}
$$

When quarks are coupled to $(N+1)^{2}$ gauge singlets, the scalar potential is given by (2.7) with $V_{L}$ being the potential of the massive $S P(N)$ theory with $N+1$ flavors. $V_{S}=0$ is not compatible with the quantum constraint and SUSY is broken [3, 4 . When $N$ flavors are given mass, while the last flavor is coupled to a gauge singlet, $\mathrm{S}$, the meson vev's scale as:

$$
M_{i j} \sim S^{\frac{1}{N+1}}, \quad M_{2 N+1,2 N+2} \sim S^{-\frac{N}{N+1}}
$$

where $i, j=1 \ldots 2 N$, and the energy vanishes as $S \rightarrow \infty$.

\section{3. $\mathbf{S U}(\mathbf{N}-\mathbf{1}) \times \mathbf{S P}\left(\frac{\mathbf{N}}{2}\right)$ versus $\mathbf{S U}(\mathbf{N}-\mathbf{1}) \times \mathbf{S U}(\mathbf{N})$ model}

Our next example will be a model described in [3] and based on $S U(N-1) \times S P\left(\frac{N}{2}\right)$ gauge group 1 with matter transforming as $Q(N-1, N), L(1, N), \bar{Q}_{i}(\overline{N-1}, 1), i=$

3 Substituting $m_{N N}=<S>$.

4 Note that we shifted $N$ relative to definition in [3], and $N$ is even. 
$1 \ldots N$. The tree level superpotential of this model is given by:

$$
W_{\text {tree }}=\lambda Q L \bar{Q}_{2}+\frac{1}{M} \sum_{i, j>2}^{N} \lambda_{i j} Q^{2} \bar{Q}_{i} \bar{Q}_{j} .
$$

This superpotential leaves classical flat directions associated with the $S U(N-1)$ antibaryons $\bar{B}=\bar{Q}^{N-1}=v^{N-1}$ (we will denote the antiquark vev by $v$ ). There is also a non-perturbative superpotential国

$$
W_{n p}=\frac{\operatorname{det} \hat{q}-\bar{B} \hat{q} B}{\Lambda_{1}^{2 N-3}}+A\left(\operatorname{Pf}(M)-\Lambda_{1}^{2 N-3} \Lambda_{2}^{N+3}\right),
$$

where $\hat{q}_{i}=q_{i}=Q \bar{Q}_{i}, q_{N+1}=L, q_{N+2}=B=Q^{N-1}, \bar{B}=\bar{Q}^{N-1}$, and $M_{i j}=q_{i} q_{j}$.

First, consider the limit $\Lambda_{1} \gg \Lambda_{2}$. SU $(N-1)$ confines and below the scale $\Lambda_{1}$ the effective theory is $S P\left(\frac{N}{2}\right)$ with $N_{f}=\frac{N}{2}+1$ flavors. The correct description of physics is given in terms of the following canonically normalized fields

$$
q_{i}=\frac{Q \bar{Q}_{i}}{\Lambda_{1}}, q_{N+1}=L, q_{N+2}=\frac{B}{\Lambda_{1}^{N-2}}=\frac{Q^{N-1}}{\Lambda_{1}^{N-2}}, S=\frac{\bar{B}}{\Lambda_{1}^{N-2}}=\frac{\bar{Q}^{N-1}}{\Lambda_{1}^{N-2}} .
$$

The superpotential of equations (3.1) and (3.2) turns into

$$
W=\sum_{i j>1}^{N+1} m_{i j} q_{i} q_{j}+\frac{P f^{\prime}(M)}{\Lambda_{1}^{N-3}}-S_{i} q_{i} q_{N+2}+\tilde{A}\left(P f(M)-\tilde{\Lambda}_{2}^{N+2}\right) .
$$

Here $m_{2, N+1}=\lambda \Lambda_{1}, m_{i j}=\frac{\lambda_{i j} \Lambda_{1}^{2}}{M}, \tilde{\Lambda}_{2}^{N+2}=\frac{\Lambda_{2}^{N+3}}{\Lambda_{1}}, M_{i j}=q_{i} q_{j}, \tilde{A}=\Lambda_{1}^{2 N-2} A$, and $P f^{\prime}$ denotes the Pfaffian over the first $\frac{N}{2}$ flavors. Without loss of generality we can set $S_{i}=0$, $M_{i, N+2}=0$ for $i \neq 1$ and be left with the model described in the previous section (with the change $N \rightarrow \frac{N}{2}$ ). We conclude that there is a run-away direction $S \rightarrow \infty$ along which scalar potential goes to zero:

$$
V_{S}=\left|\frac{\partial W}{\partial S}\right|^{2} \sim S^{-\frac{2 N}{N+2}}
$$

This solution, however, is only valid for $S \ll \Lambda_{1}$. analyze the behavior of the model at scales $S \sim \Lambda_{1}$. At scales $S \gg \Lambda_{1}$ the relevant degrees

5 We will denote $\Lambda_{S U(N-1)}$ as $\Lambda_{1}$, and both $\Lambda_{S P\left(\frac{N}{2}\right)}$ and later $\Lambda_{S U(N)}$ as $\Lambda_{2}$.

6 We thank Scott Thomas for discussion of this point. 
of freedom are the elementary ones. We still can make use of our previous results. Namely, as long as $V_{L}$ is exactly zero in terms of meson degrees of freedom, it will be zero in terms of quark degrees of freedom. Therefore, we only have to reconsider behavior of $V_{S}$ :

$$
V_{S}=\left|\frac{\partial W}{\partial S} \frac{\partial S}{\partial \bar{Q}}\right|^{2}=(N-1)^{2}\left|M_{1, N+2}\right|^{2} v^{2(N-2)} \sim v^{2 \frac{N-4}{N+2}}
$$

We see that for $N>4$ it increases along the classical flat direction. Thus the classical flat direction is stabilized quantum mechanically. Analysis of the theory in the finite region of the field space [3] shows that SUSY is broken. It is interesting to note that neglecting the quantum modified constraint in $S P\left(\frac{N}{2}\right)$ group (second term in (3.2)) one can find supersymmetric minima with vev's of the fields of order $\Lambda_{1}$. However, these minima are incompatible with the quantum modified constraint.

For $N=4$ (that is for the $S U(3) \times S P(2)$ model) the potential is constant independent of moduli. The situation is somewhat analogous to the example in $\S 2$. The $\beta$-function for the renormalizable coupling in eqn. (3.1) is

$$
\beta_{\lambda}=\frac{8}{16 \pi^{2}} \lambda^{3}-\frac{\lambda}{16 \pi^{2}}\left(\frac{16}{3} g_{1}^{2}+5 g_{2}^{2}\right)
$$

If $\lambda$ is chosen so that right-hand side of (3.7) is positive, it grows with the scale. In the limit $g_{1} \gg g_{2}$ there is a fixed point for the ratio $\lambda / g_{1}$. If $\lambda$ is chosen sufficiently small it is bounded from above by its fixed point value and is, therefore, asymptotically free. Analysis of the non-renormalizable terms is more complicated and requires at least some assumptions about the properties of the underlying microscopic theory. Nevertheless, the general conclusion is that corrections to the Kähler potential may or may not lead to run-away behavior depending on the choice of the parameters of the model.

We would like to compare these results to the behavior of the model [7] based on $S U(N-1) \times S U(N)$ gauge group with matter in the fundamental representations: $Q(N-$ $1, N), \bar{L}_{a}(1, N)$, and $\bar{Q}_{i}(\overline{N-1}, 1)$, where $a=1 \ldots N-1$, and $I=1 \ldots N$. The tree level superpotential is given by:

$$
W_{\text {tree }}=\sum_{i a} \lambda_{i a} Q \bar{L}_{a} \bar{Q}_{i}
$$


As in the previous case there are classical flat directions parameterized by $S U(N-1)$ antibaryons. The non-perturbative superpotential is given by

$$
W_{n p}=\frac{\operatorname{det}(q)-B q \bar{B}}{\Lambda_{1}^{2 N-3}}+A\left(\operatorname{det}(q \bar{q})-\operatorname{det}(q) \operatorname{det}(\bar{q})-\Lambda_{1}{ }^{2 N-3} \Lambda_{2}{ }^{2 N+1}\right),
$$

where $q=Q \bar{Q}, B=\bar{q}_{N}=Q^{N-1}, \bar{B}=\bar{Q}^{N-1}$, and, finally, $\bar{q}_{a}=L_{a}$. Let us comment on the similarities of the two models. Both of them can be constructed in the following way. Start with $S U(N-1)$ gauge group and $\mathrm{N}$ flavors of matter in the fundamental representation and gauge a subgroup of the global symmetry (one also needs to add matter transforming with quantum numbers of the second gauge group to cancel anomalies). In both models the $S U(N-1)$ gauge group is confining, it also has a flat direction parameterized by the antibaryons. The second gauge group is one flavor short of developing a quantum constraint (2.1) or (2.12). Such a constraint develops in the effective theory upon confinement of $S U(N-1)$. Therefore we can expect a similar behavior.

In fact this is true for $S=\frac{\bar{B}}{\Lambda_{1}^{N-2}} \ll \Lambda_{1}$. As in the Intriligator - Thomas model, the $S U(N-1)$ group confines and the effective theory is $S U(N)$ with $N$ flavors. $N-1$ of them are massive, while the last flavor is coupled to a $S U(N)$ singlet field. Using (2.11) we find for $S \ll \Lambda_{1}$

$$
V_{S}=\left|\frac{\partial W}{\partial S}\right|^{2} \sim S^{-2 \frac{N-1}{N}}
$$

in complete analogy with (3.5). On the other hand above the $S U(N-1)$ confinement scale result is qualitatively different

$$
V_{S}=(N-1)^{2}\left|M_{N N}\right|^{2} v^{2(N-2)} \sim v^{-\frac{2}{N}} .
$$

There is still a run-away direction in the full quantum theoryl

We saw that classical flat directions are stabilized due to the special group structure and matter content of the Intriligator-Thomas model. It is easy to repeat this analysis in the limit $\Lambda_{1} \ll \Lambda_{2}$. Then we would find exactly the same results as before but stabilization of the classical flat direction would appear to be a consequence of the presence of the nonrenormalizable terms in the $S U(N-1) \times S P\left(\frac{N}{2}\right)$ model.

7 It is possible to add non-renormalizable operators to the superpotential lifting remaining classical flat directions. In such a case SUSY is broken [7]. 


\section{4. $\mathrm{SU}(\mathrm{N}-\mathrm{M}) \times \mathrm{SU}(\mathrm{N})$ models}

A simple generalization [8] of the $S U(N-1) \times S U(N)$ model of the previous section is based on $S U(N-M) \times S U(N)$ gauge group with the following matter content: $Q(N-$ $M, N), \bar{Q}_{i}(\overline{N-M}, 1)$, and $\bar{L}_{a}(1, \bar{N})$, where $I=1 \ldots N, a=1 \ldots N-M$. The tree level superpotential is given by:

$$
W_{\text {tree }}=\lambda_{i a} Q \bar{L}_{a} \bar{Q}_{i}
$$

This lifts all flat directions except those associated with the $S U(N-M)$ antibaryons $\bar{B}^{i_{1} \ldots i_{M}}=\left(\bar{Q}^{N-M}\right)^{i_{1} \ldots i_{M}}$. Along the classical flat directions the $S U(N)$ gauge group remains unbroken and its quantum effects have to be taken into account. $S U(N)$ gauge dynamics generates a non-perturbative superpotential:

$$
W_{n p}=\left(\frac{\Lambda^{2 N+M}}{\operatorname{det}(Q \bar{L})}\right)^{\frac{1}{M}}
$$

This results in a scalar potential which can be written as

$$
V=V_{L}+V_{\bar{Q}}
$$

where $V_{L}$ is the scalar potential of massive SQCD with $N-M$ flavors and $\bar{Q}$ vev's acting as masses, while

$$
V_{\bar{Q}}=\left|\frac{\partial W}{\partial \bar{Q}_{i}}\right|^{2} .
$$

Again using (2.3) we can easily see that when $V_{L}=0$ and $\bar{B} \sim \bar{Q}^{N-M} \rightarrow \infty$, the second term in (4.3) is also vanishing: $V_{\bar{Q}} \rightarrow 0$. SUSY, therefore, remains unbroken 1 .

It is possible to construct a dual for the $S U(N-M) \times S U(N)$ model. It is instructive to see how SUSY is restored in the dual picture. The dual gauge group is $S U(M) \times S U(N)$ with fields transforming as $q(M, \bar{N}), \bar{q}_{i}(\bar{M}, 1), M_{i}(1, N), \bar{L}_{a}(1, \bar{N}), I=1 \ldots N, a=$ $1 \ldots N-M$.

8 As in the $S U(N-1) \times S U(N)$ model it is possible to add non-renormalizable terms to the superpotential (4.1). At least for $M=2$ all flat directions can be lifted. Then for odd $N$ supersymmetry is broken [8]. 
The tree level superpotential consists of the terms inherited from the electric theory as well as terms added in the process of dualizing 9

$$
W_{\text {tree }}=\lambda_{a i} \mu M_{i} \bar{L}_{a}+M_{i} q \bar{q}_{i}
$$

This superpotential leaves flat directions parameterized by the $S U(M)$ antibaryons $\bar{q}^{M} \sim$ $\bar{Q}^{N-M}$, which are exactly the flat directions of the electric theory. In the dual description $S U(N)$ has $N$ flavors, and therefore, develops a quantum constraint 10

$$
W_{n p}=A\left(\operatorname{det}(q M)-b \bar{b}-\tilde{\Lambda}^{2 N}\right)
$$

where $b=M^{N}, \bar{b}=q^{N}$, and $\tilde{\Lambda}$ is the scale of the dual theory. Now we can use (2.3) and (2.7) (remember that $\bar{q}_{i}$ are $S U(N)$ singlets) to conclude that the run-away behavior persists in the quantum theory, and that the vacuum energy vanishes as $\bar{B} \rightarrow \infty$. This conclusion remains valid above $\tilde{\Lambda}$.

One clarification is needed here. The above discussion assumes that at least in one description model is weakly coupled and the Kähler potential is nearly canonical along the classical flat direction. This is not always true. Suppose that we chose $M$ so that both original $S U(N-M)$ and its dual $S U(M)$ groups are asymptotically free. Neglecting the $S U(N)$ dynamics the model has a fixed point where both descriptions are strongly coupled. However, the scaling of the Kähler potential at the fixed point is known, thus strong coupling effects can be taking into account, and one finds that model still exhibits run-away behavior! 1 .

\section{Some Other Models}

In this section we will briefly discuss models for which a solution to $V_{L}=0$ does not exist. As an example we will use the $S U(N) \times S U(4) \times U(1)$ model of ref. [6] with the field content given by

$$
A(\boxminus, 1)_{8}, \quad a(1, \boxminus)_{-2 N}, \quad T(\square, \square)_{4-N}, \quad \bar{F}_{i}(\bar{\square}, 1)_{-4}, \quad \bar{Q}_{i}(1, \bar{\square})_{N},
$$

\footnotetext{
9 The parameter $\mu$ appearing here relates scales of electric and magnetic theories.

10 We will use here a notation $q_{M+a}=\bar{L}_{a}$.

11 We thank M. Peskin for the discussion of this point
} 
where $i=1 \ldots N$. All classical flat directions of this model are lifted if the tree-level superpotential is chosen to be

$$
\begin{array}{r}
W_{\text {tree }}=A \bar{F}_{1} \bar{F}_{2}+A \bar{F}_{3} \bar{F}_{4} \ldots+A \bar{F}_{N-2} \bar{F}_{N-1}+a \bar{Q}_{2} \bar{Q}_{3}+ \\
a \bar{Q}_{4} \bar{Q}_{4}+\ldots+a \bar{Q}_{N-1} \bar{Q}_{1}+T \bar{F}_{1} \bar{Q}_{1}+\ldots+T \bar{F}_{N} \bar{Q}_{N} .
\end{array}
$$

We will follow [6] and set coefficients of the terms $a \bar{Q}_{i} \bar{Q}_{j}$ to zero. Then the model possesses classical flat directions. Along the most general flat direction the $S U(4)$ gauge group is completely broken while the $S U(N)$ gauge group remains unbroken. Three flavors of the $S U(N)$ fields become massive and the low energy effective theory has $S U(N)$ gauge group with antisymmetric tensor, $N-4$ antifundamentals, and tree-level superpotential which raises all the flat directions of the "reduced" theory. Such a model is known to break supersymmetry 12 with the vacuum energy

$$
V \sim \Lambda_{L}^{4} \sim\left(v^{4} \Lambda^{2 N-1}\right)^{\frac{4}{2 N+3}}
$$

where $v$ denotes generic vev of the moduli. We immediately see that a potential for the moduli is generated, and there is no run-away behavior. An analysis of the strong coupling dynamics near the origin of the moduli space conducted in [6] shows that supersymmetry is broken in the model. Analogous conclusions apply to the $S U(N) \times S U(3) \times U(1)$ and $S U(N) \times S U(5) \times U(1)$ models of [5, 6].

\section{Conclusions}

In this note we considered the behavior of models with classical flat directions. We showed that it is convenient to analyze physics along these directions in two stages. First, one considers the moduli as fixed parameters. If the "reduced" theory breaks SUSY, and its scale increases with the moduli vev, then classical flat directions are stabilized. If the scale decreases then theory exhibits run-away behavior. If the "reduced" theory does not break SUSY one has to consider dynamics for the moduli subject to the requirement

12 In general one has to investigate in this way all possible flat directions. In this case if we chose to look in a more specialized flat direction the low energy effective theory would be different, but still supersymmetry breaking. 
that potential of the "reduced" theory vanishes. At different scales the theory may be best described by different sets of variables. Therefore this analysis should be repeated at all relevant scales. The asymptotic behavior of the potential can be determined by the analysis in the region where moduli vev greatly exceed any dynamically generated scale of the model. This means that the answer can usually be found most easily from an analysis in terms of the elementary degrees of freedom.

In some cases one finds pseudo-flat direction even in the presence of a non-perturbative superpotential. Then corrections to the Kähler potential determine the location of the true vacuum. We showed that in certain regimes such corrections are calculable and can be computed as renormalizations of the Yukawa couplings. Thus the potential at infinity is determined by asymptotic behavior of the coupling constants of the model. In the Intriligator-Thomas $S U(2)$ the non-asymptotically free nature of the Yukawa coupling lead to the stabilization of the potential. Moreover, we showed that for sufficiently small coupling $\lambda$ there are (calculable) minima for large but finite values of the moduli fields. We also saw an example where the potential could be destabilized due to the existence of a quasi-fixed point for the Yukawa coupling.

Acknowledgments. We would like to thank Michael Dine, and Scott Thomas for useful discussions and reading of the manuscript. We would also like to thank Michael Peskin for useful discussions. This work was supported in part by the U. S. Department of Energy. 


\section{References}

[1] I. Affleck, M. Dine, and N. Seiberg, Nucl. Phys. B256 (1985) 557.

[2] K. Intriligator, S. Seiberg, S. Shenker, Phys. Lett. 342B (1995) 152, hep-ph/94102203; M. Dine, A. Nelson, Y. Nir, Y. Shirman, Phys. Rev. D53 (1996) 2658, hep-th/9507378; E. Poppitz, S. Trivedi, Phys. Lett. 365B (1996) 125, hep-th/9507169; P. Pouliot, Phys. Lett. 367B (1996) 151, hep-th/9510148; P. Pouliot, M. Strassler, Phys. Lett. 375B (1996) 175, hep-th/9602031; A. Nelson, Phys. Lett. 369B (1996) 277, hep-th/9511350; C.-L. Chou, hep-th/9605108; C. Csaki, W. Skiba, M. Schmaltz, hep-th/9607210; K. Intriligator, S. Thomas, hep-th/9608046.

[3] K. Intriligator, S. Thomas, hep-th/9603158.

[4] K. Izawa, T. Yanagida, Prog. Theor. Phys. 95 (1996) 829, hep-th/9602180.

[5] C. Csaki, L. Randall, W. Skiba, hep-th/9605108.

[6] C. Csaki, L. Randall, W. Skiba, R. Leigh, hep-th/9607021.

[7] E. Poppitz, Y. Shadmi, S. Trivedi, hep-th/9606184.

[8] E. Poppitz, Y. Shadmi, S. Trivedi, hep-th/9605113.

[9] N. Seiberg, Phys. Rev. D49 (94) 6857, hep-th/9402044; K. Intriligator, N. Seiberg hep-th/9509066.

[10] K. Intriligator, P. Pouliot, Phys. Lett. 353B (1995) 471, hep-th/9506006. 